\title{
Understanding the formation of apremilast cocrystals
}

Marta K. Dudek, ${ }^{1,2}$ Ewelina Wielgus, ${ }^{1}$ Piotr Paluch, ${ }^{1}$ Justyna Śniechowska, ${ }^{1}$ Maciej Kostrzewa, ${ }^{1}$ Graeme M. Day, ${ }^{2}$ Grzegorz D. Bujacz, ${ }^{3}$ and Marek J. Potrzebowski ${ }^{1}$

${ }^{1}$ Centre of Molecular and Macromolecular Studies PAS, Sienkiewicza 112, 90363 Lodz, Poland

${ }^{2}$ Computational Systems Chemistry, School of Chemistry, University of Southampton, SO17 1BJ, UK

${ }^{3}$ Institute of Technical Biochemistry, Technical University of Lodz, Stefanowskiego 4/10, 90924 Lodz, Poland

\begin{abstract}
Apremilast (APR), an anti-psoriatic agent, easily forms isostructural cocrystals and solvates with aromatic entities, often disobeying at the same time Kitaigorodsky's rule as to the saturation of possible hydrogen bonding sites. In this paper the reasons standing behind this peculiar behavior are investigated, employing a joint experimental and theoretical approach. This includes the design of cocrystals with coformers having high propensity towards the formation of both aromatic - aromatic and hydrogen bonding interactions, determination of their structure, using solid state NMR spectroscopy and X-Ray crystallography, as well as calculations of stabilization energies of formation of the obtained cocrystals, followed by crystal structure prediction calculations and solubility measurements. Our findings indicate that the stabilization energies of cocrystal formation are positive in all cases, which results from strain in the APR conformation in these crystal forms. On the other hand, solubility measurements show that the Gibbs free energy of formation of the apremilast : picolinamide cocrystal is negative, suggesting that the formation of the studied cocrystals is entropy-driven. This entropic stabilization is associated with the disorder observed in almost all known cocrystals and solvates of APR.
\end{abstract}

\section{Introduction}

Pharmaceutical cocrystals are usually defined as entities with at least two different solid components confined in a single crystal lattice, with at least one of these components being an active pharmaceutical ingredient, API, whereas the other one being either another API, or, more frequently, a substance from the generally-regarded-as-safe, GRAS, list (Bolla \& Nangia, 
2016). The components of a cocrystal are held together by non-covalent interactions, most frequently by hydrogen bonds, but also by halogen bonds or aromatic - aromatic interactions (Mukherjee \& Desiraju, 2014; Wicker et al., 2017). A significant increase of scientific interest in their formation and stability can be explained by the fact that cocrystals, just like any new solid crystal form, can often offer better physicochemical properties, including solubility, thermal stability and bioavailability (Karki et al., 2009; Douroumis et al., 2017; Grifasi et al., 2015; Kaur et al., 2017). For example, the formation of cocrystals of glibenclamide (Goyal et al., 2018), epalrestat (Putra et al., 2017), quercetin (Vasisht et al., 2016), and cilostazol (Yoshimura et al., 2017) has been recently proven to be profitable in enhancing water solubility of an API, when compared with the respective neat solid form.

One API with poor water solubility and short shelf-life is apremilast (APR), a phosphodiesterase-4 inhibitor used in psoriasis and psoriatic arthritis (Schafer, 2012). Its commercially available form B, characterized only by its powder X-Ray diffractogram, is regarded as the most thermodynamically stable one (US Patent 9850205B2, 2017), but suffers from the above mentioned unfavorable physicochemical properties (Shakeel et al., 2017). It is not surprising, therefore, that new forms are sought after, and so far a number of better soluble isostructural solvates and cocrystals has been reported (Wu et al., 2017a; Wu et al., 2017b; Dudek et al., 2018; Wang et al., 2018; European Patent EP3339292A1, 2018). One of the most characteristic and unique features of these forms is the fact, that they are held together almost entirely by aromatic - aromatic interactions, even though the solvent and/or coformer molecules used for their formation, as well as APR itself, are capable of forming strong hydrogen bonds (Dudek et al., 2018; Wang et al., 2018).

A detailed analysis of functional groups present in APR indicates that, apart from the two sites engaged in an intramolecular hydrogen bond, i.e. $\mathrm{CO}$ and $\mathrm{NH}$ amide groups, there are at least 6 sites with strong propensity of acting as hydrogen bond acceptors: two oxygen atoms from the sulfonyl group, two methoxy oxygen atoms, and two carbonyl groups (Figure 1). A survey of the sulfonyl group propensity towards the formation of hydrogen bonds has shown that in all of 12 analyzed cases oxygen atoms from sulfonyl group were hydrogen bond acceptors, while in 10 cases methylene protons acted as hydrogen bond donors (Brondel et al., 2010). Similarly, it has been demonstrated that oxygen from the methoxy group in methoxybenzenes is a strong acceptor of hydrogen bonds from various donor groups (Palusiak \& Grabowski, 2002), not to mention the carbonyl oxygen from isoindoline-1,3-dione ring, which often acts as a hydrogen bond acceptor for $\mathrm{C}-\mathrm{H}$ aromatic protons. Meanwhile, in APR cocrystals and solvates none of these hydrogen bond donors and acceptors are involved in the 
formation of such a bond, be this either with another APR molecule or a coformer (Wu et al., 2017a; Dudek et al., 2018). Clearly, certain factors capable of compensating the observed deviation from the maximum saturation of hydrogen bonds trend upon formation of cocrystals (described as early as in 1973 by Kitaigorodsky) have to exist. We have recently shown that there is a strong preference for $\pi-\pi$ interactions over hydrogen bond formation in the cocrystals and solvates of APR, and that it can be exploited to form a variety of cocrystals with pharmaceutically acceptable coformers (Dudek et al., 2018), but the reason standing behind this preference remains unclear. This paper aims at clarifying this preference and explaining the factors that influence such a marked violation of Kitaigorodsky's rule. To that purpose we account for three possibilities: (i) exceptionally favorable energy of aromatic - aromatic interactions in APR cocrystals, (ii) an existence of a yet unknown stable crystal structure of APR cocrystals held together by hydrogen bonds, and (iii) a stabilizing role of disorder present in almost every known isostructural solvate and cocrystal of APR. As coformers, four different aromatic compounds were selected: resorcinol, picolinamide, imidazole, and hydroquinone, all being capable of acting as hydrogen bond donors, and all exhibiting a preference towards hydrogen bonds formation.

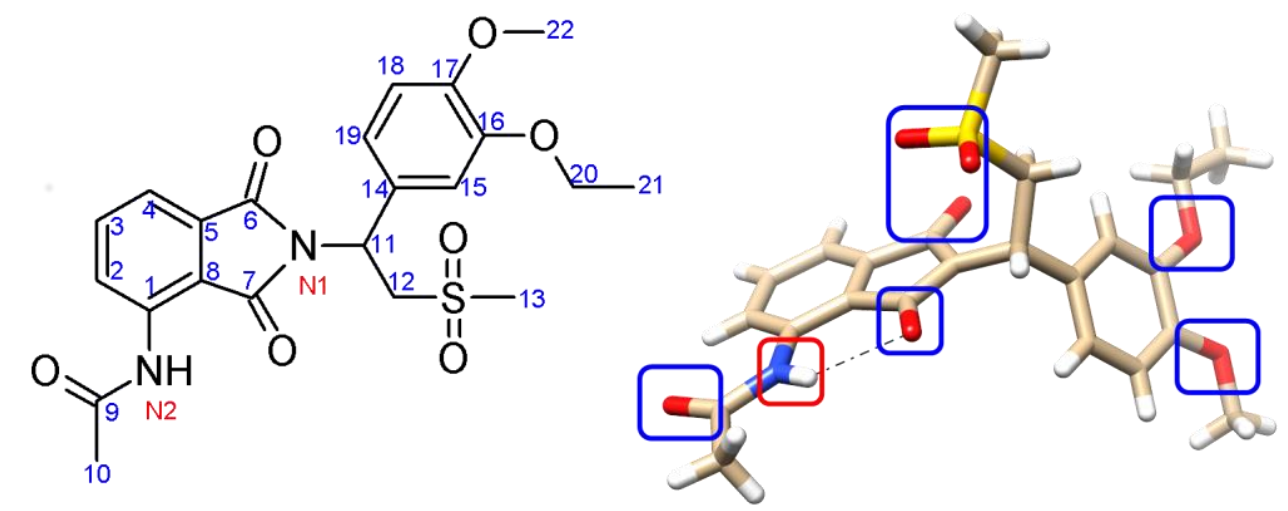

Figure 1. Structure of APR with numbering and hydrogen bond (HB) sites marked with blue rectangles (HB acceptors), and with a red rectangle (HB donor).

\section{Experimental Section}

\section{Materials}

APR ((S)-N-(2-[1-(3-ethoxy-4-methoxyphenyl)-2-methane sulfonylethyl]-1,3-dioxo-2,3-dihydro-1H-isoindol-4-yl) acetamide) was purchased from Accel Pharmtech company and was recrystallized from acetone (POCh) via very slow evaporation of solvent to obtain suitable crystals of form B, which was identified by the comparison of the PXRD pattern (see Supporting Information, Figure S2) with patent literature data. Resorcinol (RES, Sigma 
Aldrich), hydroquinone (HQU, Sigma Aldrich), picolinamide (PIC, TCI Chemicals) and imidazole (IMI, Merck) were used as is.

\section{Cocrystal formation}

Cocrystals of APR were synthesized using a mechanochemical approach, which for the purpose of obtaining suitable crystals for single crystal X-Ray diffraction studies, was followed by solution recrystallization. In both cases the identity of the resulting crystalline forms was evaluated with ${ }^{13} \mathrm{C}$ CPMAS NMR experiments. In the mechanochemical approach $100 \mathrm{mg}$ of APR was ground for $1 \mathrm{~h}$ in a ball mill set to $25 \mathrm{~Hz}$ frequency with an appropriate amount of a coformer, so that the resulting molar ratio was equal to 1:0.5. In all cases 20 ul of water was added to the reaction mixture to give liquid assisted grinding (LAG) conditions. All grinding experiments resulted in appropriate cocrystals formation, which was examined via solid-state NMR measurements. In order to obtain suitable crystals for single crystal X-Ray measurements ethyl acetate (STANLAB) was used to recrystallize cocrystals obtained via mechanochemistry. Note that direct crystallization of pure APR and appropriate coformers from ethyl acetate end up in the formation of ethyl acetate APR solvate. In the case of APR:IMI cocrystals, we noticed that during grinding and crystallization experiments, a new, unknown polymorphic form appeared in small quantities. The crystal structure determination of this new form is beyond the scope of this manuscript and will be evaluated in our future work.

\section{Single crystal X-Ray measurements}

Single crystal diffraction experiments for form B of apremilast, as well as for the four cocrystals were carried out on Oxford SuperNova single-crystal diffractometer with micro source $\mathrm{Cu} \mathrm{K \alpha}$ radiation $(\lambda=1.5418 \AA)$ with a Titan detector. Diffraction data collection, cell refinement and data reduction were performed using the CrysAlis PRO program (Oxford Diffraction). The structure was solved by direct methods SHELXS implemented in OLEX2 package (Dolomanov et al., 2009) and refined using full-matrix least-squares methods SHELXL (Sheldrick, 2015). The crystal structures were deposited at the CSD under the deposition numbers 1916487, 1916491, 1916488, 1916489 and 1916490 for apremilast form B, APR:RES, APR:PIC, APR:IMI and APR:HQU, respectively.

\section{Solid-state NMR experiments}

${ }^{13} \mathrm{C}$ and ${ }^{15} \mathrm{~N}$ CPMAS experiments have been performed with a Bruker Avance II 400 spectrometer equipped with a $4 \mathrm{~mm}$ probe-head, operating at $400.13,100.90$ and $40.56 \mathrm{MHz}$ 
frequencies for ${ }^{1} \mathrm{H},{ }^{13} \mathrm{C}$ and ${ }^{15} \mathrm{~N}$, respectively. Samples were spun at $8 \mathrm{kHz}$ and a standard Bruker CP pulse programs with $2 \mathrm{~ms}$ contact time and spinal decoupling during acquisition were used. ${ }^{1} \mathrm{H}-{ }^{13} \mathrm{C}$ inverse detected HETCOR and ${ }^{1} \mathrm{H}-{ }^{1} \mathrm{H}$ Back-to-Back correlation experiments were carried out on a Bruker Avance III 600 spectrometer equipped with $1.3 \mathrm{~mm}{ }^{1} \mathrm{H} /{ }^{13} \mathrm{C} /{ }^{15} \mathrm{~N}$ triple resonance CP-MAS probe-heads with the ${ }^{1} \mathrm{H},{ }^{13} \mathrm{C}$ resonance frequencies of 600.13 and $150.90 \mathrm{MHz}$ respectively. In all proton detected experiments samples were spun at $60 \mathrm{kHz}$.

Inverse detected HETCOR experiment have been carried out employing sequence described by Mao et al. (2009) and Althaus et al. (2014), using a RF equal to $100 \mathrm{kHz}$ on ${ }^{13} \mathrm{C}$ channel during contact time. For ${ }^{1} \mathrm{H}$ linear ramp from 90 to $100 \%$ with maximum RF value near to $160 \mathrm{kHz}$ have been used. Actual RF value of ${ }^{1} \mathrm{H}$ RF during first and second contact have been precisely optimized on individual samples. Unwanted ${ }^{1} \mathrm{H}$ magnetization from ${ }^{12} \mathrm{C}$ isotopologues have been canceled out by applying two long $(20 \mathrm{~ms})$ pulses with phase $\mathrm{X}$ and $\mathrm{Y}$ and RF equal to $30 \mathrm{kHz}$ (HORROR condition) (Nieslen et al., 1994). Low power ${ }^{1} \mathrm{H}$ decoupling during $\mathrm{t} 1$ evolution and low power ${ }^{13} \mathrm{C}$ decoupling during acquisition have been applied with an RF equal in both cases to $10 \mathrm{kHz}$ applying swftpp decoupling sequence. For initial setup $\mathrm{U}-{ }^{13} \mathrm{C},{ }^{15} \mathrm{~N}-l$ histidine hydrochloride monohydrate have been used. First contact time had always duration equal to $2 \mathrm{~ms}$. Two experiments have been carried for individual samples with second contact time equal to $100 \mathrm{us}$ and $1 \mathrm{~ms}$. In the ${ }^{1} \mathrm{H}-{ }^{1} \mathrm{H}$ Back-to-Back experiments DQ excitation as well as reconversion time was $33.3 \mu \mathrm{s}$, while $\mathrm{t} 1$ increment was equal to $16.67 \mathrm{us} .{ }^{13} \mathrm{C}$ chemical shifts have been referenced according to secondary standard adamantane. ${ }^{1} \mathrm{H}$ chemical shifts have been referenced according to solid sample of $\mathrm{d}_{4}$-TSP (methyl signal at $0 \mathrm{ppm}$ ).

\section{Powder X-Ray diffraction experiments}

Powder diffraction experiments were performed using a borosilicate glass capillary $(0.5 \mathrm{~mm}$ diameter) and a Bruker D8 Discover powder diffractometer equipped with a sealed copper tube ( $\mathrm{Cu}$ K $\alpha$ radiation, $\lambda=1.5406 \AA$, formed by Goebel mirror monochromator). The registered data range was $5-50^{\circ} 2 \theta$, with a step of $0.05^{\circ}$.

\section{Quantum chemical calculations}

CASTEP calculations. For the assignment of the NMR parameters of form B of APR electronic structure calculations under periodic boundary conditions were performed for the obtained crystal structure with the CASTEP code (Clark et al., 2005), using the PBE functional with D2 dispersion correction scheme (Grimme, 2006), ultrasoft pseudopotentials, plane wave energy cut-off of $600 \mathrm{eV}$, and $0.07 \AA^{-1}$ separation to sample k-points in a Brillouin zone. All these parameters were primarily tested for convergence. In geometry optimization all atomic 
positions were allowed to relax, while keeping cell parameters rigid. After successful optimization NMR parameters were calculated, with a GIPAW approach (Yates et al., 2007) and the same level of theory as stated above. The theoretical shieldings were subsequently used together with the experimental NMR data (2D correlations) to assign all the ${ }^{1} \mathrm{H},{ }^{13} \mathrm{C}$ and ${ }^{15} \mathrm{~N}$ resonances.

To evaluate the energy of cocrystal formation, geometry optimization at the same level of theory was performed for the crystal structures of the studied coformers and for the obtained cocrystals. In the case of the cocrystals, due to the fact that there are two equally probable possible positions of coformers, for each cocrystal two structures, each with one of the possible positions of a coformer were geometry optimized, and mean energy was used in all the following calculations. Subsequently, single point energy calculations were carried out for each symmetry unrelated molecule of APR from form B, as well as from cocrystals, and for each of the coformers placed in a $20 \AA$ box, in order to calculate inter- and intramolecular energy contributions. To calculate the stabilization energies for the studied cocrystals $\left(\mathrm{E}^{\mathrm{stab}}\right)$, the following equations were used:

$E^{\text {stab }}=E_{\text {cocr }}^{\text {total }}-\left(n E_{A P R}^{\text {total }}+m E_{\text {coformer }}^{\text {total }}\right)$

$\Delta^{\text {intra }}=E_{\text {cocr }}^{\text {intra }}-E_{A P R}^{\text {intra }}$

$\Delta^{\text {inter }}=\left(E_{\text {cocr }}^{\text {total }}-\sum_{\text {comp }} k * E_{\text {comp }}^{\text {intra }}\right)-\left(E_{\text {pure }}^{\text {total }}-\sum k * E_{\text {pure }}^{\text {intra }}\right)$

where $E_{\text {cocr }}^{\text {total }}, E_{A P R}^{\text {total }}, E_{\text {coformer }}^{\text {total }}$ denote total energies for a cocrystal, pure form B of APR and pure coformer, respectively, while $\Delta^{\text {intra }}$ and $\Delta^{\text {inter }}$ are the intra- and intermolecular energy contribution terms to the stabilization energy, calculated as differences between these terms obtained for cocrystals and pure forms, and $n, m$ and $k$ values denote number of molecules accounted for in a crystal, reflecting the ratio of particular components.

Gaussian calculations. Molecular electrostatic potential (MEP) surfaces were evaluated at the B3LYP-GD3BJ/6-311G(d,p) level of theory with Gaussian09 code (Frisch et al., 2009), using molecular geometries from the optimized crystal structures. To evaluate the energetics of aromatic - aromatic and hydrogen bonds interactions, single point energy calculations for the optimized crystal geometries of appropriate molecular clusters, and corresponding isolated components were performed at the B3LYP-GD3BJ/6-311G(d,p) level of theory with the Gaussian09 code. The clusters were built in a way so that the molecules were interacting only via one the regarded interactions. For aromatic - aromatic interactions two molecules of APR, forming a $\pi$-philic space with and without the presence of a coformer were used. Similarly, 
hydrogen-bonding interaction clusters were built. All the clustered arrangements are shown in Supporting Information. In that way an energy contribution of aromatic - aromatic and hydrogen bonds interactions to the total energy could be estimated separately, as most of the other intermolecular interactions are excluded from the system with such arrangements. Finally, the energy differences between the systems with and without the appropriate interactions were calculated in a way so that they would correspond to an asymmetric part of a unit cell in the studied cocrystals.

Crystal Structure Prediction (CSP). For CSP calculations, the optimized geometry of APR from the crystal structure of a cocrystal was used to generate trial crystal structures in three chiral space groups: $P 2{ }_{1}, P 2{ }_{1}{ }_{12}{ }_{1}$ and $P 41$, using the Global Lattice Energy Explorer code (Case et al., 2016). The intramolecular energy contribution to the total energy of the trial structures were optimized using DMACRYS 2.2.1.0 (Price et al., 2010), keeping molecular geometries rigid, and using atom-centred distributed multipoles up to hexadecapoles, calculated from the charge density obtained from Gaussian calculations using GDMA 2.2.11 (Stone, 2005). For repulsion-dispersion interactions, the FIT potential (Coombes et al., 1996) with a $25 \AA$ cut-off was used. In each of the space groups the calculations were continued until 50000 successfully geometry optimized structures have been found. Subsequently, the structures were clustered on the basis of their PXRD pattern, energy and density similarities to remove duplicates.

\section{Solubility measurements}

The concentration of APR for solubility measurements of APR:PIC cocrystals and physical mixture was determined by ACQUITY UPLC I-Class chromatography system coupled with SYNAPT G2-Si mass spectrometer equipped with an electrospray source and quadrupoleTime-of-Flight mass analyser (Waters Corp., Milford, MA, USA). Acquity BEНтм C18 column $(100 \times 2.1 \mathrm{~mm}, 1.7 \mu \mathrm{m})$ maintained at $40^{\circ} \mathrm{C}$ temperature was used for the chromatographic separation of analyte. A gradient program was employed with the mobile phase, combining solvent A (0.1\% formic acid in water) and solvent B (acetonitrile) as follows: $20 \%$ B (0-0.1 $\min ), 20-95 \%$ B (0.1-2.3 min), 95-95\% B (2.3-3.0 min), 95-20\% B (3.0-3.1 min) and 20$20 \% \mathrm{~B}(3.1-4$. min). The flow rate was $0.40 \mathrm{~mL} / \mathrm{min}$, and the injection volume was $1 \mu \mathrm{L}$.

For mass spectrometric detection the electrospray source was operated in a negative resolution mode. The optimized source parameters were: capillary voltage $2.5 \mathrm{kV}$, cone voltage $20 \mathrm{~V}$, desolvation gas flow $600 \mathrm{~L} / \mathrm{h}$ with temperature $350{ }^{\circ} \mathrm{C}$, nebulizer gas pressure $6.5 \mathrm{bar}$, and source temperature $110^{\circ} \mathrm{C}$. Mass spectra were recorded over an $m / z$ range of 100 to 1200 . The 
system was controlled using MassLynx software (Version 4.1), while data processing (peak area integration, calibration curve) was performed by TargetLynxTM program.

The initial stock calibration solution of APR was created with a concentration of approximately $10 \mathrm{mg} / \mathrm{mL}$ of APR in acetonitrile. The stock solution was serial diluted (with acetonitrile) to obtain working solutions at several concentration levels. The calibration curves were prepared at six different concentrations of APR solutions and were linear over a concentration range from $10 \mathrm{ng} / \mathrm{mL}$ to $2000 \mathrm{ng} / \mathrm{mL}$ with a correlation coefficient of $>0.987$.

The samples for solubility measurement were prepared by dissolving an excess amount of each solid (APR:PIC cocrystals and a physical mixture in 1:0.5 ratio) in chloroform. The obtained suspensions were stirred at $25^{\circ} \mathrm{C}$ for 24 hours. After filtrations through a $0.22 \mu \mathrm{m}$ PTFE syringe filter the filtrates were diluted with acetonitrile to obtain appropriate concentration of solution (in the range of UPLC-MS calibration). The determined concentrations of APR were reported as the average of two replicated experiments for each sample of APR:PIC cocrystals and physical mixture and three calibration curves.

\section{Results and Discussion.}

\section{1) Structural characterization of pure APR and pure coformers.}

A first step to the understanding of cocrystals formation is the analysis of pure crystalline forms of both components. However, in the case of APR no pure crystal structure is available in the CSD database, but the patent literature indicates that APR can form at least 5 pure crystalline structures (patents no. US9850205B2, 2017; EP3339292A1, 2018; WO2009/120167A1, 2009; WO2017/196192A1, 2017). Due to the fact, that in this work commercially available form B of APR has been used in the co-crystallization experiments, and that it is regarded as the most thermodynamically stable form, we decided to put an extra effort to crystallize and solve its crystal structure.

Apremilast was crystallized from acetone by slow solvent evaporation to form needlelike (rod-like) crystals of monoclinic system with space group $P 2{ }_{1}$. The independent unit of this crystal form contains four apremilast molecules which are assembled in two dimers. The main interactions responsible for this dimer creation are $\pi$ stacking interactions of the substituted phenyl ring, with both dimers having similar architecture. Interestingly, in the solid-state NMR spectra of this form, only two to three sets of resonances are clearly distinguishable for most sites, with many ${ }^{1} \mathrm{H},{ }^{13} \mathrm{C}$ and ${ }^{15} \mathrm{~N}$ nuclei from corresponding sites in not symmetry-related molecules resonating at exactly the same frequencies (Figure 2). This is due to the presence of 
the previously mentioned dimers, each having very similar conformers of APR, with the molecular overlay RMSD for each pair being equal to 0.353 and $0.315 \AA$, respectively. These two dimers interact in a crystal mainly within themselves, forming layers held together via aromatic - aromatic interactions, while the interlayer interactions are mainly those between $\mathrm{CH}_{3}-\mathrm{C}=\mathrm{O}$ and $\mathrm{CH}_{3}-\mathrm{CH}_{3}$ groups (Figure 2). Such close resemblance of the four symmetryunrelated molecules results in poor resolution of the ${ }^{1} \mathrm{H}$ MAS NMR spectrum with broad signals due to their mutual overlap, as well as in serious difficulties in the ${ }^{1} \mathrm{H}$ and ${ }^{13} \mathrm{C}$ resonances assignments to respective molecules, despite registering the spectra under very fast MAS conditions (that is with the spinning speed of $60 \mathrm{kHz}$ ). Here, to do the exact assignment, NMR calculations under periodic boundary conditions were necessary. The assigned resonances, together with ${ }^{1} \mathrm{H}$ and 2D spectra are given in Supporting Information, while all crystallographic data are shown in Tables 1 and $\mathrm{S} 1$.

Table 1. Crystallographic data for form $B$ of APR, as well as for APR:RES, APR:PIC, APR:IMI and APR:HQU cocrystals.

\begin{tabular}{|c|c|c|c|c|c|}
\hline Compound & APR:RES & APR:PIC & APR:IMI & APR:HQU & APR form $B$ \\
\hline & $\mathrm{C}_{22} \mathrm{H}_{24} \mathrm{~N}_{2} \mathrm{O}_{7} \mathrm{~S}$ & $\mathrm{C}_{22} \mathrm{H}_{24} \mathrm{~N}_{2} \mathrm{O}_{7} \mathrm{~S}$, & $\mathrm{C}_{22} \mathrm{H}_{24} \mathrm{~N}_{2} \mathrm{O}_{7} \mathrm{~S}$ & $\mathrm{C}_{22} \mathrm{H}_{24} \mathrm{~N}_{2} \mathrm{O}_{7} \mathrm{~S}$ & $\mathrm{C}_{22} \mathrm{H}_{24} \mathrm{~N}_{2} \mathrm{O}_{7}$ \\
\hline Empirical & , & $0.5\left(\mathrm{C}_{6} \mathrm{H}_{6} \mathrm{~N}_{2} \mathrm{O}\right.$ & , & , & S \\
\hline formula & $0.5\left(\mathrm{C}_{6} \mathrm{H}_{6} \mathrm{O}_{2}\right)$ & ) & $0.5\left(\mathrm{C}_{3} \mathrm{H}_{4} \mathrm{~N}_{2}\right)$ & $0.5\left(\mathrm{C}_{6} \mathrm{H}_{6} \mathrm{O}_{2}\right)$ & \\
\hline & $0.5\left(\mathrm{H}_{2} \mathrm{O}\right)$ & & $0.5\left(\mathrm{H}_{2} \mathrm{O}\right)$ & $0.5\left(\mathrm{H}_{2} \mathrm{O}\right)$ & \\
\hline $\begin{array}{c}\text { Formula } \\
\text { weight }\end{array}$ & 524.55 & 521.55 & 501.52 & 524.55 & 460.49 \\
\hline $\begin{array}{c}\text { Temperatur } \\
\text { e }\end{array}$ & $100 \mathrm{~K}$ & $100 \mathrm{~K}$ & $100 \mathrm{~K}$ & $100 \mathrm{~K}$ & $293 \mathrm{~K}$ \\
\hline $\begin{array}{l}\text { Crystal } \\
\text { system }\end{array}$ & tetragonal & tetragonal & tetragonal & tetragonal & monoclinic \\
\hline Space group & $P 4{ }_{12}{ }_{12}$ & $P 4{ }_{12}{ }_{12}$ & $P 4{ }_{12}{ }_{12}$ & $P 4{ }_{12}{ }_{12}$ & $P 21$ \\
\hline $\mathrm{a}(\AA)$ & $12.89179(7)$ & $12.81222(8)$ & $12.85808(14)$ & $12.88747(7)$ & $8.71383(13)$ \\
\hline $\mathrm{b}(\AA)$ & $12.89179(7)$ & $12.81222(8)$ & $12.85808(14)$ & $12.88747(7)$ & $29.4960(6)$ \\
\hline$c(\AA)$ & $29.5087(3)$ & $29.7274(3)$ & $29.5715(4)$ & $29.5246(3)$ & $17.6717(2)$ \\
\hline
\end{tabular}




$\begin{array}{cccccc}\alpha\left(^{\circ}\right) & 90 & 90 & 90 & 90 & 90 \\ \beta\left(^{\circ}\right) & 90 & 90 & 90 & 90 & 97.9370(14) \\ \gamma\left({ }^{\circ}\right) & 90 & 90 & 90 & 90 & 90\end{array}$

Volume

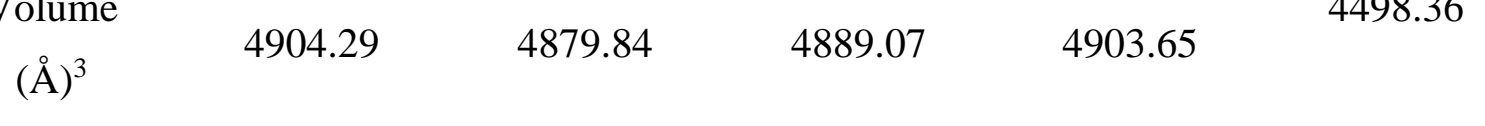

Density $\left(\mathrm{g} / \mathrm{cm}^{3}\right)$

$\begin{array}{llll}1.421 & 1.420 & 1.363 & 1.421\end{array}$

$\begin{array}{lllllll}\mathrm{Z} & 8 & 8 & 8 & 8 & 8\end{array}$

$R$ value 0.047 0.058 0.063 0.060 0.045
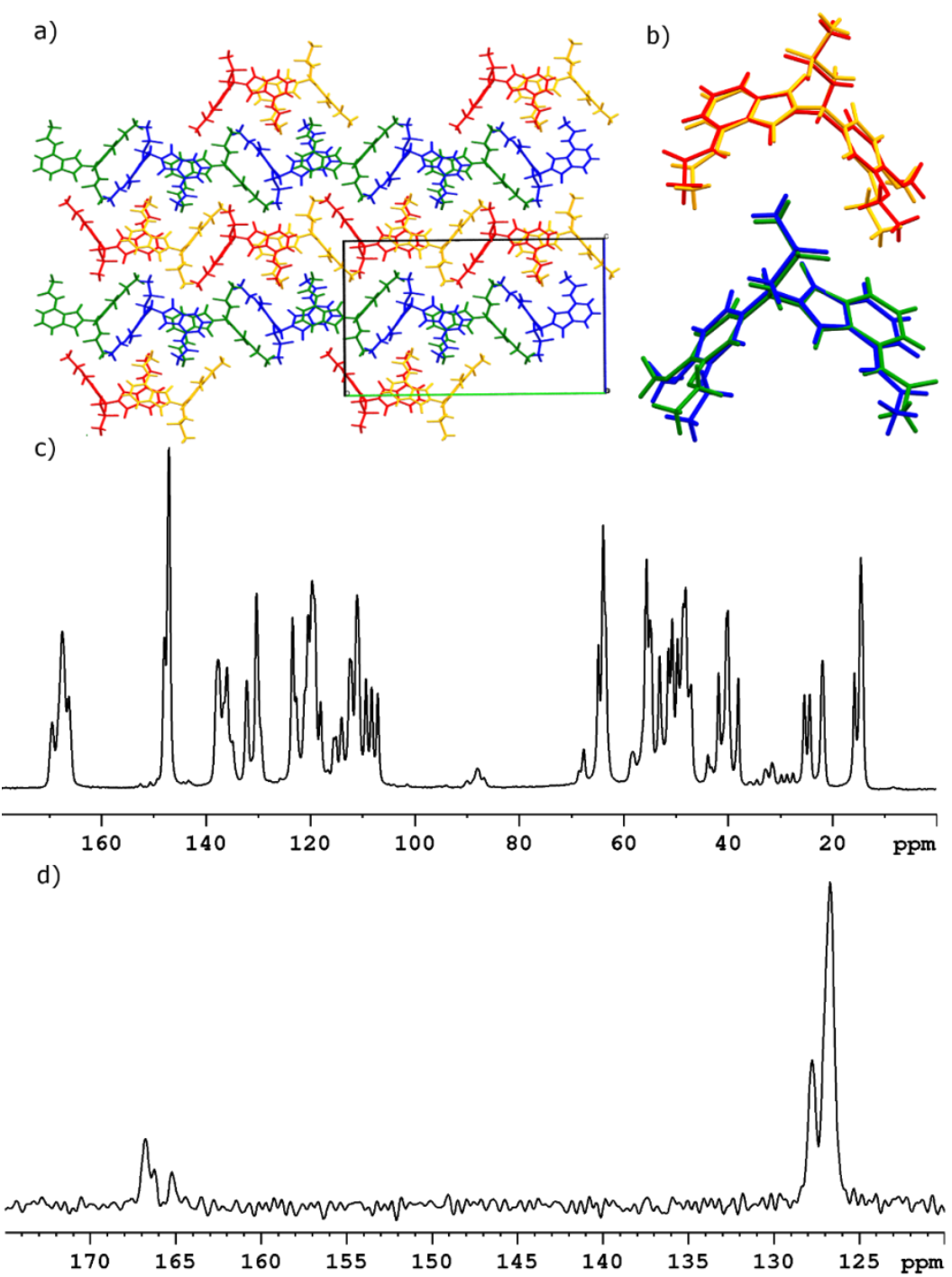
Figure 2. Structural data obtained for form B of APR: crystal packing (a) and molecular overlays of two pairs of symmetry unrelated molecules (b) observed in the crystal, ${ }^{13} \mathrm{C}$ and ${ }^{15} \mathrm{~N}$ solid-state NMR spectra (c and d, respectively). Colors of APR molecules denote symmetryunrelated molecules.

In the case of coformers used in this study, all of them already have known crystal structures. Resorcinol and hydroquinone are closely related positional isomers, but they exhibit somewhat different crystallization preferences. For resorcinol there are three known crystal forms, $\alpha, \beta$, and $\varepsilon$ (with RESORA CSD refcode) (Bacon \& Jude, 1973; Bacon \& Lisher, 1980; Zhu et al., 1026), two having $P$ na $2{ }_{1}$ and one $P 2{ }_{1} 2{ }_{1}{ }_{1}$ space group symmetry. Similarly, hydroquinone crystallizes in one of the three known crystal forms: $\alpha, \beta$, and $\gamma$ (all having refcodes starting with HYQUIN) (Maartmann-Moe, 1966; Lindeman et al., 1981), with P21/c, $P 3$ and $R-3$ space group symmetry, respectively. In each of these crystal forms both hydroxyl groups are engaged each in two hydrogen bonds: one as a donor and one as an acceptor of the bond. Also in picolinamide (refcode PICAMD) (Evora et al., 2012), as well as in imidazole crystals (refcode IMAZOL) (Craven et al., 1977), hydrogen bonds govern the crystal packing of these molecules (Figure 3). Our choice of coformers for the preparation of APR cocrystals was based on their tendency to form both hydrogen bonds and aromatic - aromatic interactions. However, as can be seen from the data presented above and in Figure 3, in all crystals of the selected coformers aromatic - aromatic interactions are either not observed, or arranged in such a way as to not distort the hydrogen bonding pattern. Contrarily, in APR molecules mainly aromatic - aromatic interactions govern the observed packing.

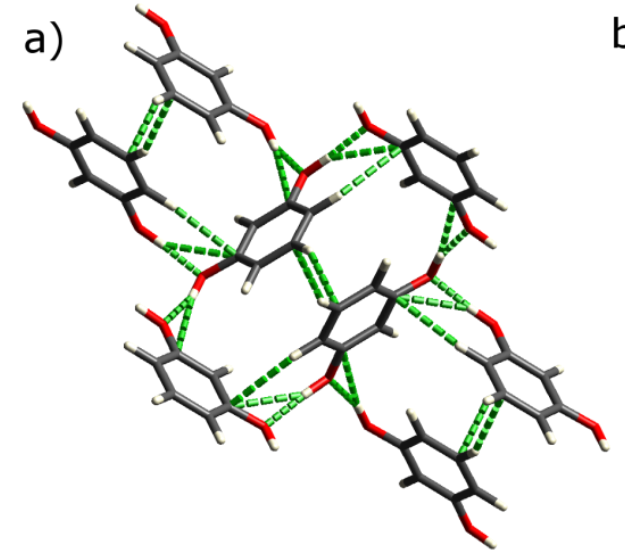

b)

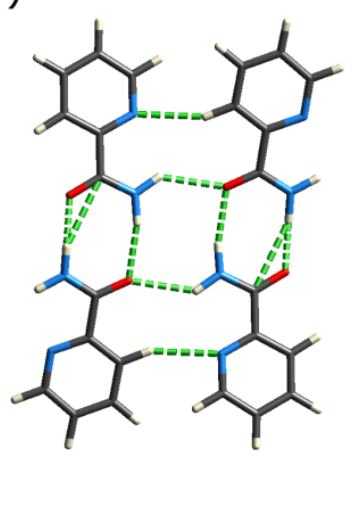

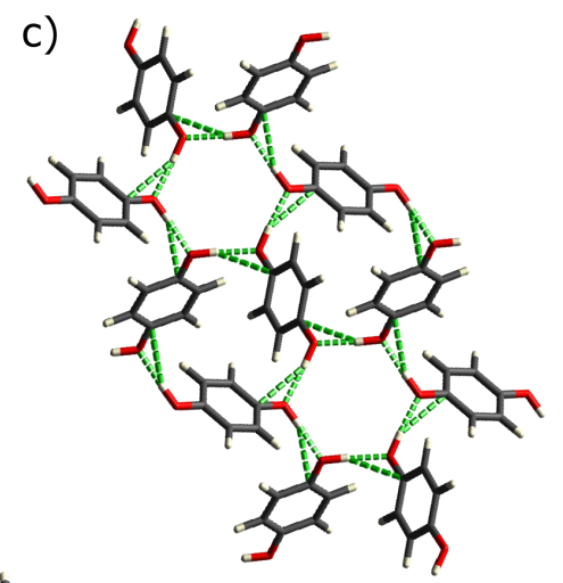

d)

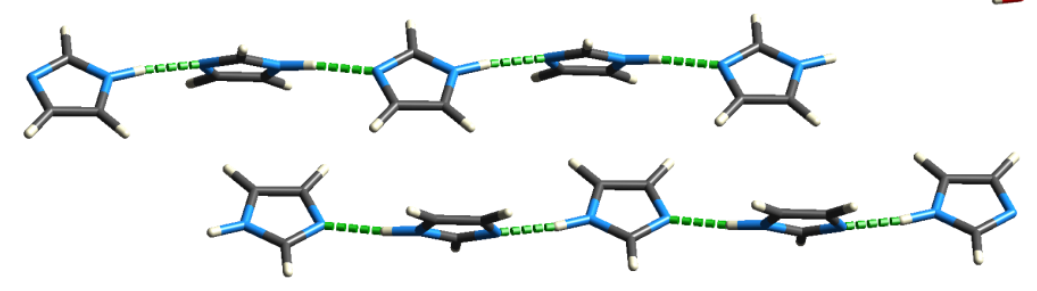


Figure 3. Hydrogen bonding motifs in neat crystals of coformers used in this study: $\alpha$-resorcinol (a), picolinamide (b), $\alpha$-hydroquinone (c), and imidazole (d).

\section{2) Structural characterization of APR cocrystals.}

Despite strong propensity of coformers to form hydrogen bonds, definitely outweighing their tendency to interact via aromatic - aromatic interactions, as indicated by the analysis of their pure crystal structures, all four cocrystals formed by them with APR are isostructural and held together mostly by the aromatic - aromatic interactions. This can be concluded from a very similar outlook of their ${ }^{13} \mathrm{C}$ CPMAS NMR spectra, very characteristic for systems with specific sandwich-like arrangement of molecules of APR and coformers (Figures S3 and S5, Supporting Information). In all the spectra the ${ }^{13} \mathrm{C}$ signals assignable to coformers carbon atoms can be distinguished, with ca. 50\% smaller signals intensities, as compared to those originating from APR, which reflects 2:1 APR:coformer ratio found in all APR solvates and cocrystals published so far. On the other hand, their ${ }^{1} \mathrm{H}$ MAS spectra, as well as ${ }^{1} \mathrm{H}-{ }^{1} \mathrm{H}$ Back-to-Back (BaBa) correlation spectra are different to a certain extent (Figure 4), reflecting possible differences in the intermolecular interactions and close contacts of the ${ }^{1} \mathrm{H}$ atoms.

a)
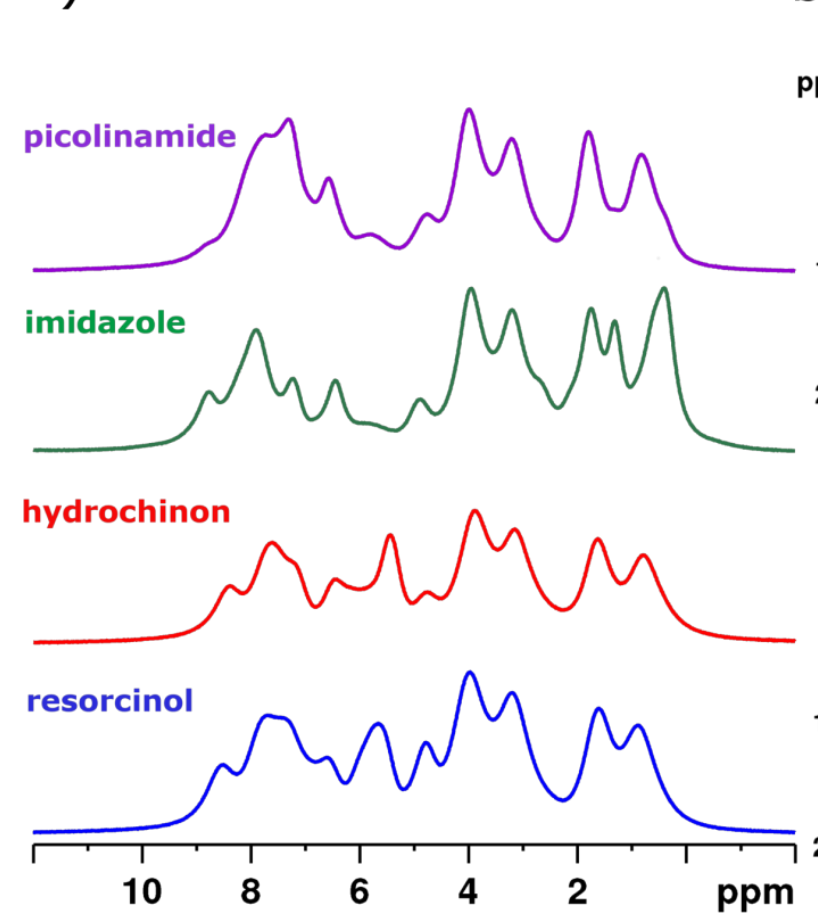

b)
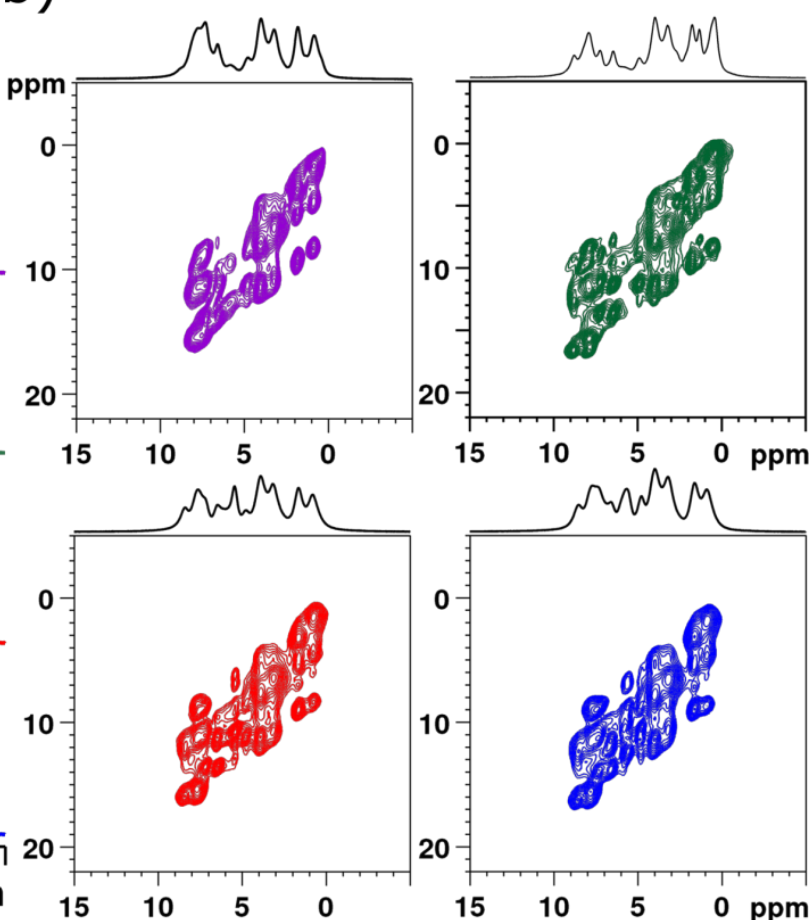

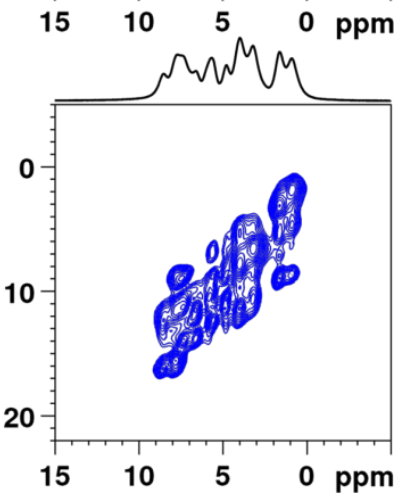

Figure 4. ${ }^{1} \mathrm{H}$ VF MAS NMR spectra recorded with spinning rate $60 \mathrm{kHz}$ for the cocrystals of APR with different coformers: ${ }^{1} \mathrm{H}$ MAS NMR spectra (a), and SQ-DQ BaBa 2D NMR spectra (b) registered at $60 \mathrm{kHz}$ spinning speed. Colors used for spectra plotting denote particular coformers. 
A closer look at the differences in ${ }^{1} \mathrm{H}$ chemical shifts between the studied cocrystals (Table 2) reveals that the most prominent changes concern $\mathrm{H} 2$ and $\mathrm{H} 3$ atoms, with noticeably lower values found for a cocrystal with PIC, as well as NH hydrogen atoms from amide group of APR, which are similar for cocrystals with RES and HQU, but not so for the other cocrystals. As it is not likely for the intramolecular hydrogen bond of this $\mathrm{NH}$ proton with $\mathrm{CO}$ group to be broken, these latter differences may reflect a proximity of different functional groups originating from different coformers. On the other hand, the observed differences for $\mathrm{H} 2$ and H3 chemical shifts indicate somewhat similar interactions (or proximities) of APR molecule with particular coformers in IMI, RES and HQU cocrystals, distinct from those present in APR:PIC cocrystal. Not so prominent, but possibly important difference in the ${ }^{1} \mathrm{H}$ chemical shifts concerns hydrogen atoms from methylsulfonyl group, namely methyl H13. For APR:HQU cocrystal its chemical shift is lower than for all the other cocrystals. The difference is small, but in all isostructural solvates and cocrystals studied so far this particular chemical shift value is well preserved and almost always equal to ca. $3.20 \mathrm{ppm}$. Its lower value in APR:HQU cocrystal may suggest that one of the sulfonyl group oxygen atoms is engaged in the hydrogen bond with a coformer.

Table 2. ${ }^{1} \mathrm{H}$ chemical shifts of four isostructural cocrystals of APR and the studied coformers.

\begin{tabular}{lllll}
\hline atom & APR:RES & APR:HQU & APR:IMI & APR:PIC \\
\hline 2 & 8.51 & 8.43 & 8.79 & 8.08 \\
3 & 7.83 & 7.63 & 7.84 & 7.32 \\
4 & 7.76 & 7.73 & 7.90 & 7.73 \\
10 & 1.62 & 1.62 & 1.72 & 1.82 \\
11 & 4.92 & 4.78 & 4.90 & 4.82 \\
12 & $3.76+$ & $3.73+5.83$ & $3.92+$ & $3.85+5.88$ \\
13 & 6.00 & 3.13 & 5.86 & 3.22 \\
15 & 3.21 & 8.15 & 3.20 & 8.11 \\
18 & 8.14 & 7.18 & 8.05 & 7.31 \\
19 & 7.32 & 6.49 & 7.22 & 6.63 \\
20 & 6.63 & $3.43(2 \mathrm{H})$ & 6.46 & $3.47(2 \mathrm{H})$ \\
21 & $3.45(2 \mathrm{H})$ & 0.79 & $3.34(2 \mathrm{H})$ & 0.84 \\
22 & 0.91 & 3.91 & 0.56 & 4.05 \\
$\mathrm{NH}$ & 4.03 & 7.54 & 3.97 & 7.79 \\
& 7.43 & & 8.15 & \\
\hline
\end{tabular}


To follow up the conclusions drawn from solid-state NMR measurements, single crystal X-Ray diffraction experiments for the four cocrystals were performed (for crystallographic data see Table 1). Indeed, all the obtained crystals were found to be in the shape of tetragonal bipyramids, crystallizing isostructurally in the $P 4{ }_{1} 212$ tetragonal space group, with aromatic aromatic interactions playing the main role in holding together components in the crystal lattice. However, as was suggested by solid-state NMR results, some differences between these cocrystals in terms of intermolecular interactions have been found.

A first characteristic and distinctive feature is the presence of water molecule in three out of four cocrystals: APR:IMI, APR:RES and APR:HQU. In all cases water is located at the two fold axis and is strongly bonded with a coformer via a hydrogen bond, and as a consequence is located in the $\pi$-philic space formed by APR molecules, in a close proximity to $\mathrm{H}-2$ and $\mathrm{H}-3$ atoms of APR. Its presence can readily explain the higher values of ${ }^{1} \mathrm{H}$ chemical shifts observed for H-2 and H-3 atoms of these cocrystals in comparison to that of APR:PIC, with no water molecule in its structure.

As to the formation of the hydrogen bonds engaging APR molecule itself, there are only two types of them observed in the obtained structures. The first one has been found in the APR:HQU structure, and is located between one of the oxygen atoms from APR methylsulfonyl moiety and a HQU hydroxyl proton, not engaged in the hydrogen bond with water (Figure 5a). Again, this is in agreement with the suggestion from the solid-state NMR results, according to which a small, but unusual change in the ${ }^{1} \mathrm{H}$ chemical shift of the $\mathrm{CH}_{3}$ group from methylsulfonyl moiety indicated some differences in the local environment of this group. The second hydrogen bond motif is present in the three cocrystals containing water molecule, i.e. APR:RES, APR:HQU and APR:IMI. It is a bifurcated bond between water and an oxygen atom from either ethoxy or methoxy group (Figure 5b). There are no intermolecular hydrogen bonds observed in the APR:PIC structure. It is clear, that even coformers with significant hydrogen bond formation propensity did not break the tendency of APR to form an arrangement in which aromatic - aromatic interactions are promoted, even though it left some of the coformer sites capable of acting as hydrogen bond donors unbounded.

Apart from saturating one of the hydrogen bonding sites in the three coformers, interaction with water also changes the molecular electrostatic potential (MEP) surfaces of the coformers, thus creating a possibility of stronger aromatic - aromatic interaction. Figure 6 and 7 show such surfaces mapped onto electron density isosurfaces for the APR (Figure 6), and coformers with and without the presence of water molecule (if it is present in the respective crystal structures) (Figure 7). From the analysis of the APR MEP surface, it can be said that the 
isoindoline-1,3-dione ring of APR, which is engaged in the aromatic - aromatic interactions in APR cocrystals with aromatic species, can be regarded as an electron deficient aromatic ring. Indeed, it has only one site with a small negative electrostatic potential value (meaning a slightly repulsive reaction towards the negatively charged species), while all the other sites of this ring exhibit positive electrostatic potential of up to $+126 \mathrm{~kJ} / \mathrm{mol}$, indicating a strong attraction propensity towards the negative charge.

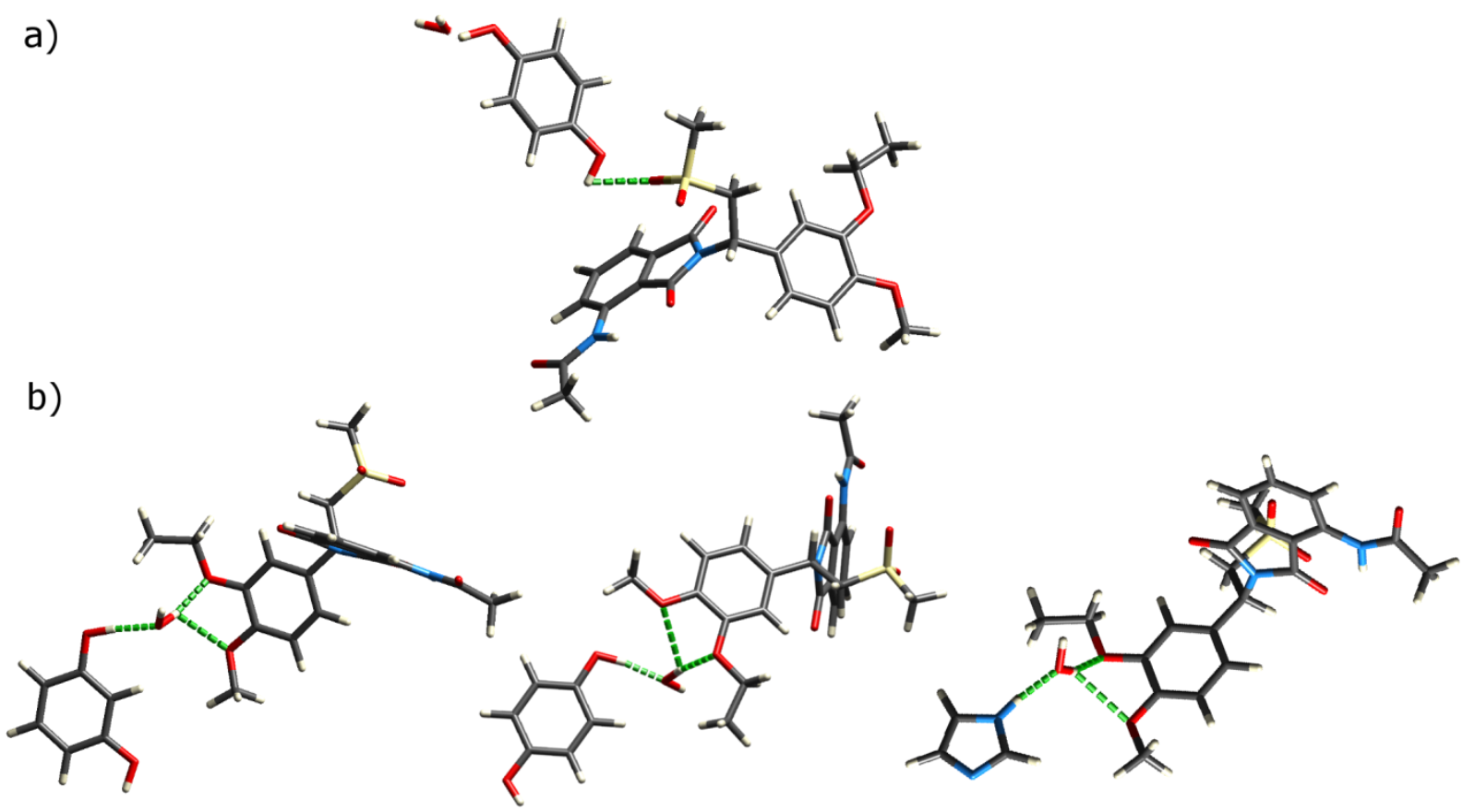

Figure 5. Two hydrogen bond motifs observed in the cocrystals of APR and the studied coformers present in: (a) APR:HQU cocrystal $\left(\mathrm{CH}_{3}-\mathrm{SO}_{2} \ldots \mathrm{OH}-\mathrm{Ar}\right)$; (b) APR:RES, APR:HQU and APR:IMI cocrystals (HO-H...OMe/OEt)

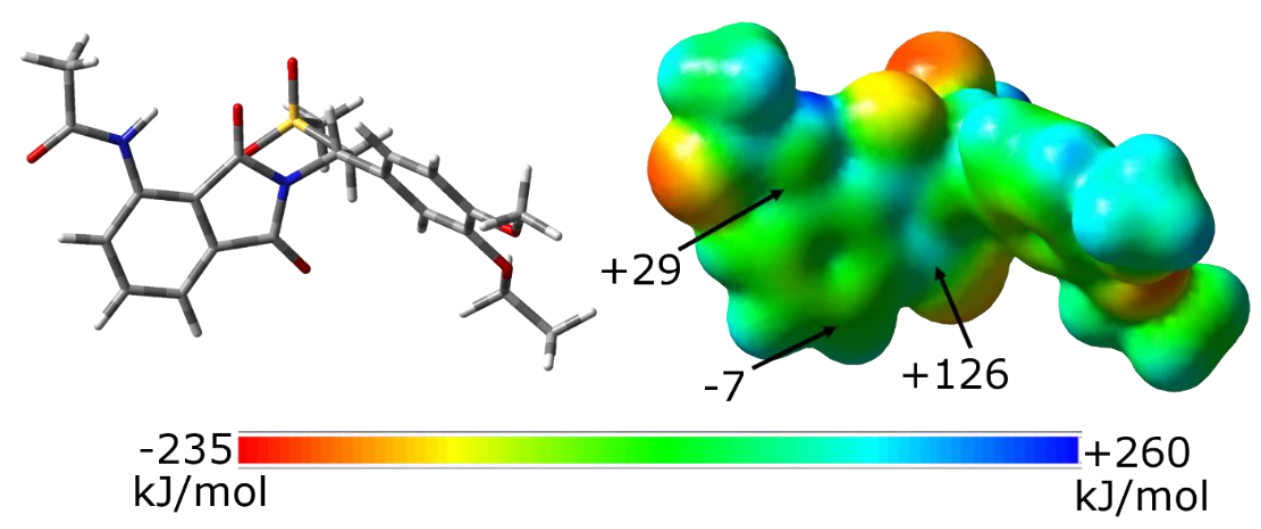

Figure 6. APR molecule viewed from the site engaged in the aromatic - aromatic interactions in the obtained cocrystals, and its molecular electrostatic potential mapped on the total electron density isosurface (0.005 a.u.). All electrostatic potential values are given in $\mathrm{kJ} / \mathrm{mol}$, MEP surfaces were calculated at the B3LYP-GD3BJ/6-311G(d,p) level of theory. 
In the case of coformer molecules, RES, IMI and HQU are all electron-rich aromatic systems, which, upon hydrogen bonding to a water molecule, become even more enriched with a negative charge, leading consequently to a stronger attraction to the electron-deficient sites of isoindoline-1,3-dione ring of APR. The only coformer molecule for which there is no water present in the cocrystal of APR is PIC. However, even without the presence of water the electrostatic potential is already sufficiently negative to be inclined to interact with the electrondeficient aromatic systems.

A

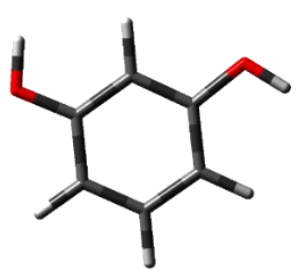

+
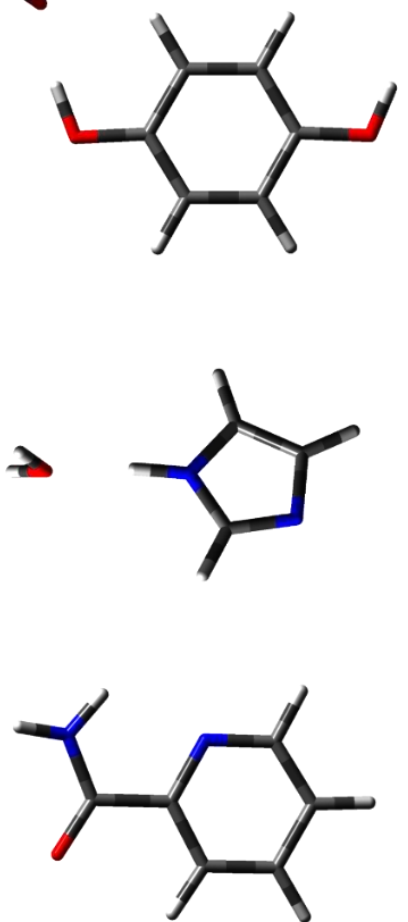

B
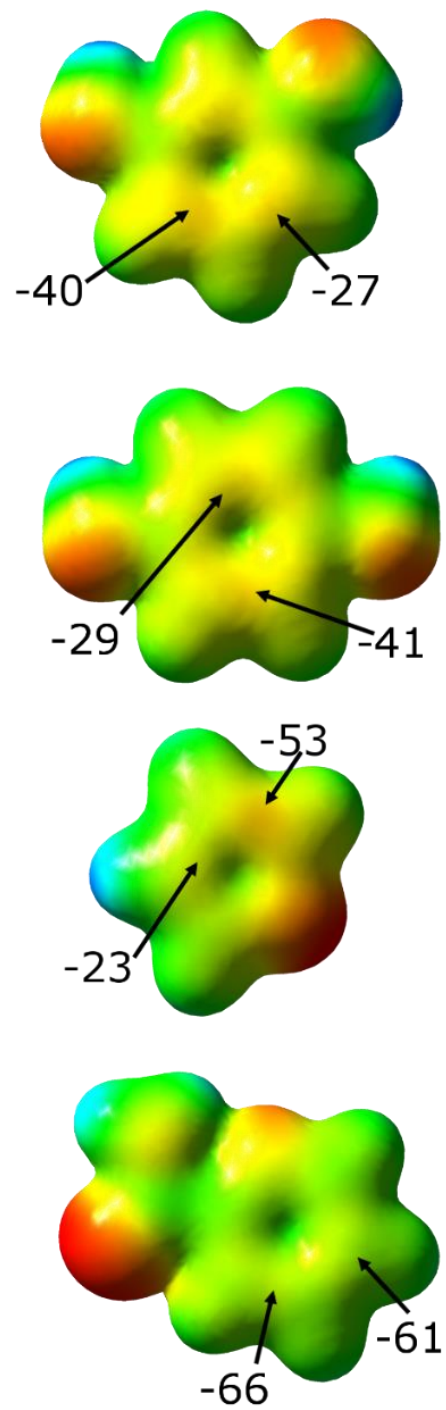

$\mathbf{C}$
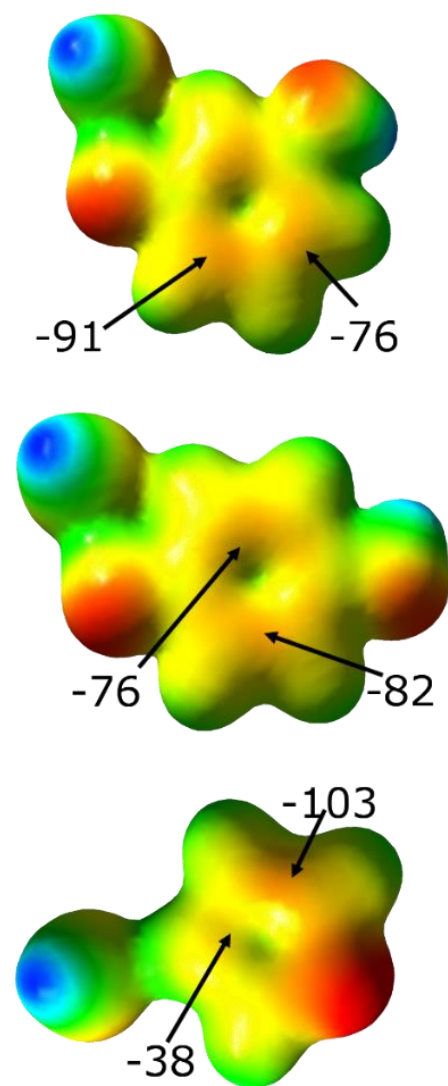

$-235$

$\mathrm{kJ} / \mathrm{mol}$

$\mathrm{kJ} / \mathrm{mol}$

Figure 7. Molecular electrostatic potential surfaces mapped on the total electron density isosurfaces (0.01 a.u.) for coformers used in this study to form cocrystals with APR without (column B) and with the presence of water (column C). Column A indicates conformations of the coformers, which in all cases are derived from the respective cocrystals. All electrostatic 
potentials are given in $\mathrm{kJ} / \mathrm{mol}$, MEP surfaces were calculated at the B3LYP-GD3BJ/6$311 \mathrm{G}(\mathrm{d}, \mathrm{p})$ level of theory.

3) Evaluation of crystal energy landscape in the search for hydrogen bonded cocrystals of APR:PIC

Among the studied cocrystals, APR:PIC is the only system for which no intermolecular hydrogen bonds have been found, despite the ability of both components to form such bonds. Therefore, crystal structure prediction (CSP) calculations were used to gain an insight into whether alternative crystal packing motifs may be energetically plausible, apart for the one found experimentally. Due to the large size of the system, the search was carried out for only three space groups $P 2{ }_{1}, P 2{ }_{1}{ }_{1}{ }_{1}$ (which are the two most common chiral space groups describing $75 \%$ of all enantiomeric organic molecules) and $P 4_{1}$. The $P 2{ }_{1} 2_{12}{ }_{1}$ and $P 4_{1}$ space groups are subgroups of $P 4{ }_{1} 2{ }_{1} 2$ space group, in which the experimental crystal structure of APR:PIC was found. Figure 8 shows the resulting CSP landscape, and features one particularly stable crystal structure, which was found to be in an excellent agreement with the experimental structure; an overlay of a 15-molecule cluster from predicted and observed crystal structures leads to a root mean squared deviation in atomic positions of only $0.118 \AA$. The CSP search that has been performed cannot be regarded as a having sampled all possible co-crystal arrangements, due to limiting the search to one conformation of APR and the most common space groups, and therefore the results have to be treated cautiously. Nevertheless, the magnitude of the energy gap between the best and the next-best structure $(76 \mathrm{~kJ} / \mathrm{mol})$ suggests that a possibility of the formation of other competitive structures is rather low. 


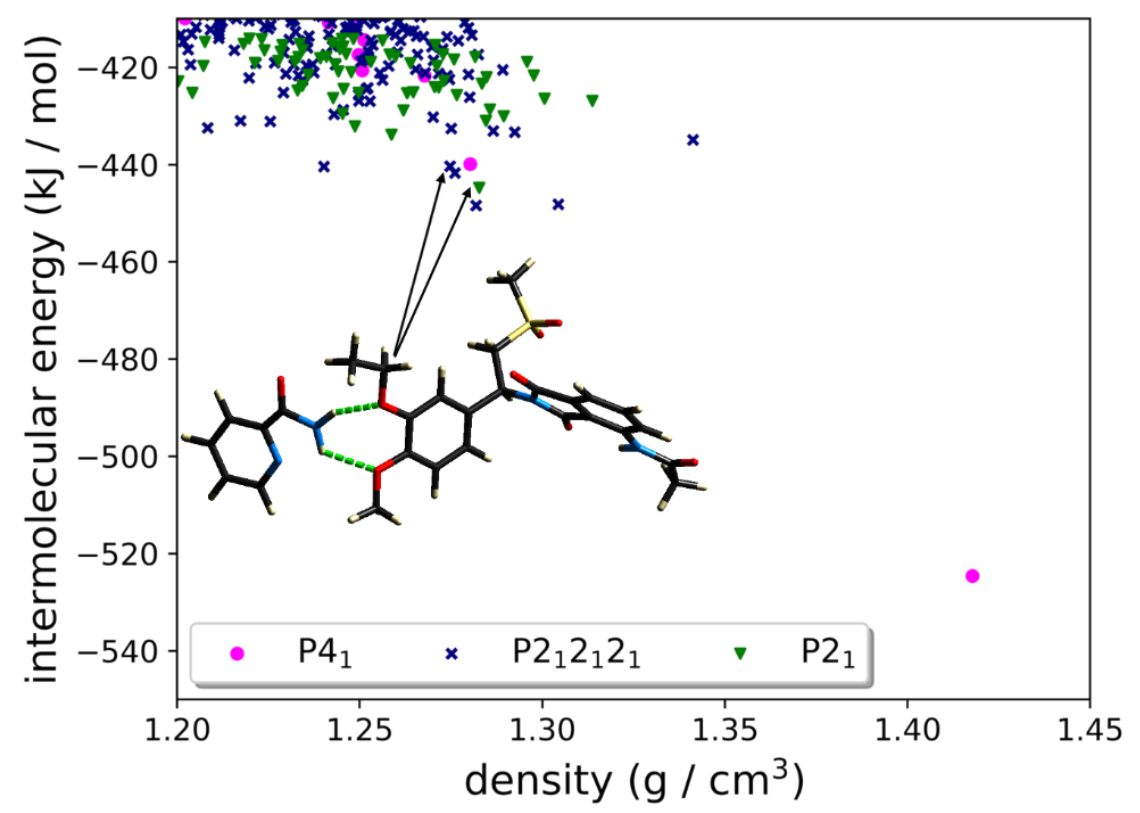

Figure 8. Crystal energy landscape for APR:PIC cocrystal tested in $P 2{ }_{1}, P 2{ }_{1}{ }_{2} 2_{1}$ and $P 4{ }_{1}$ space groups. An arrow indicates the lowest energy structure with an intermolecular hydrogen bond between APR and PIC.

An interesting feature of the predicted structures is that in $P 2{ }_{1} 2_{1} 2_{1}$ and $P 4_{1}$ space groups aromatic - aromatic interactions are preferred over hydrogen bonds. The lowest energy predicted structure in which APR and PIC interact via hydrogen bonds is in the $P 2{ }_{1}$ space group with an energy of $-445 \mathrm{~kJ} / \mathrm{mol}, 80 \mathrm{~kJ} / \mathrm{mol}$ above the lowest energy structure, followed by a structure in space group $P 212121$, with an energy of $-440 \mathrm{~kJ} / \mathrm{mol}$. Here the hydrogen bond is formed between the $\mathrm{NH}_{2}$ group from PIC and oxygen atom of the OMe and OEt groups from APR (Figure 8). Such an interaction however, results in losing the possibility of exploiting not only aromatic - aromatic interactions, but also many $\mathrm{Ar}-\mathrm{H} \ldots \mathrm{O}$ and $\mathrm{CH}_{3} \ldots \mathrm{O}$ close contacts, maintaining at the same time favorable packing efficiency.

\section{4) Evaluation of the energetic contributions to the total energy of APR cocrystals}

The X-Ray, solid-state NMR and CSP results presented above have shown that aromatic - aromatic interactions are indeed a driving force in the formation of APR cocrystals, even in cases in which a coformer displays high propensity towards acting as a hydrogen bond donor. The next step is therefore to evaluate the energetic factors behind these interactions, starting with the calculations of stabilization energies of the cocrystals, i.e. the differences between the total energy of a cocrystal and the sum of total energies of its components in their pure forms. In a recent study on the energetics of cocrystals, it has been shown that for the vast majority $(95 \%)$ of observed cocrystals, the stabilization energies have negative values, which means that 
cocrystal formation is thermodynamically driven (Taylor \& Day, 2018). Surprisingly, this seems to be not the case for the cocrystals of APR. Table 3 presents the energy difference between the energy of a cocrystal and the sum of the energies of its pure components calculated at the PBE-D2 level of theory. In all cases the stabilization energies (calculated according to eq. 1) have positive values, and this is primarily the result of noticeably higher intramolecular energies of APR molecules in the cocrystals, as compared to these energies in the pure form of APR. On the other hand, the differences in the intermolecular contributions to the total energy are in all cases negative, meaning that it is not the lack of energetically favorable interactions between APR and the coformers that makes the stabilization energies positive, but the strains in the conformation of APR associated with cocrystal formation. Regardless of the cause, it seems at this stage, that the formation of cocrystals of APR with the studied coformers is not energetically favorable.

Table 3. Overall stabilization energies $\left(E^{\text {stab }}\right)$ of the formation of cocrystals of APR with RES, HQU, IMI and PIC, and the intra- and intermolecular energy contribution to $E^{\text {stab }}$.*

\begin{tabular}{llll}
\hline cocrystal & $E^{\text {stab }}[\mathrm{kJ} / \mathrm{mol}]$ & $\Delta^{\text {inter }}[\mathrm{kJ} / \mathrm{mol}]$ & $\Delta^{\text {intra }}[\mathrm{kJ} / \mathrm{mol}]$ \\
\hline APR:RES & +3.14 & -13.37 & +16.51 \\
APR:HQU & +17.00 & -1.72 & +18.37 \\
APR:IMI & +16.79 & -0.66 & +17.44 \\
APR:PIC & +6.15 & -9.54 & +15.73
\end{tabular}

*Note, that $E^{\text {stab }}$ and $\Delta^{\text {inter }}, \Delta^{\text {intra }}$ values were calculated separately, according to equations (1-3), and as a result the two latter values add up to $E^{\text {stab }}$ only within the limits of computational errors. Note also, that intramolecular contributions from conformers were neglected.

To gain a deeper understanding of the intermolecular energy contribution of the APR cocrystals, the energetics of aromatic - aromatic and HB interactions were evaluated (Table 4). As can be seen, the energetic contributions of aromatic - aromatic interactions are equal to 50 $-60 \mathrm{~kJ} / \mathrm{mol}$ (per crystallographic asymmetric unit of a cocrystal), which is comparable to the stabilization energy typically found for strong hydrogen bonds. It is noteworthy that these interaction energies are slightly stronger for the APR:RES and APR:HQU systems, which reflects their lower electrostatic potentials found on their MEP surfaces, in comparison to the 
values found for APR:IMI and APR:PIC systems. The energy contributions from the hydrogen bonds formed between APR and coformers, excluding the interactions 'inside' the coformer (i.e. between water molecule and an aromatic entity of a coformer), are half as large (as calculated per crystallographic asymmetric unit cell), but become comparable to the $\mathrm{Ar}-\mathrm{Ar}$ contributions after including HB interactions within the coformers. It should be noted, however, that these two calculated contributions to the overall intermolecular energy constitute in all cases only one third of the total lattice energy. Therefore, apart from these obvious intermolecular interactions, the sum of the energy contributions from weaker interactions, such as $\mathrm{CH}_{3}-$ or $\mathrm{Ar}-\mathrm{H}$ with $\mathrm{SO}_{2}, \mathrm{C}=\mathrm{O}$ or $\mathrm{OMe} / \mathrm{OEt}$ oxygen atoms, has to play an important role in the stabilization of the cocrystals. Obviously, such a sum would be very difficult to calculate, but it can be estimated by subtracting the sum of the energy contribution of the aromatic aromatic and hydrogen bonds interactions from the overall intermolecular energies of the cocrystals. The obtained values, denoted as $\mathrm{E}^{\text {other }}$, are included in Table 4.

Table 4. Energy contributions of intermolecular interactions found in APR cocrystals: aromatic - aromatic interactions (Ar-Ar), hydrogen bonds between APR and a coformer (HB_APR), hydrogen bonds "inside" a coformer, i.e. between water and an aromatic entity (HB_coformer). $E^{\text {inter }}$ denotes total intermolecular energy for each of the cocrystals, whereas $E^{\text {other }}$ is the difference between $\mathrm{E}^{\mathrm{inter}}$ and the sum of $\mathrm{E}^{\mathrm{Ar}-\mathrm{Ar}}, \mathrm{E}^{\mathrm{HB} \_\mathrm{APR}}$ and $\mathrm{E}^{\mathrm{HB}{ }_{-} \text {coformer }}$. All energy values are given in $\mathrm{kJ} / \mathrm{mol}$.

\begin{tabular}{llllll}
\hline Cocrystal & $\mathrm{E}^{\mathrm{Ar}-\mathrm{Ar}}$ & $\mathrm{E}^{\mathrm{HB} \_\mathrm{APR}}$ & $\mathrm{E}^{\mathrm{HB} \_ \text {coformer }}$ & $\mathrm{E}^{\text {inter }}$ & $\mathrm{E}^{\text {other }}$ \\
\hline APR:RES & -62 & -28 & -23 & -324 & -210 \\
APR:HQU & -58 & -39 & -21 & -314 & -197 \\
APR:IMI & -48 & -27 & -19 & -300 & -206 \\
APR:PIC & -49 & - & - & -278 & -229
\end{tabular}

\section{5) Stabilizing role of disorder?}

Though the analysis of structure and energies of the studied cocrystals performed so far indicates preference for aromatic - aromatic interactions over hydrogen bonds, it does not answer the most important question: why the cocrystals are formed at all, despite positive stabilization energies? An answer to this may be provided by an evaluation of the entropy contribution to the Gibbs free energy of the cocrystals. Usually, when comparing energetic stability of crystals, their free energies are approximated by their enthalpies, and the entropic contribution to the free energy, $\Delta G=\Delta H-T \Delta S$, is neglected. However, in some cases this 
entropy contribution may play an important role in the stabilization of a crystal structure, especially in the crystals in which disorder is observed.

As mentioned before, in almost all known APR cocrystals and solvates (except for dichloromethane solvate) (Wu et al., 2017b), a coformer or a solvent molecule is disordered, even at $100 \mathrm{~K}$. The nature of this disorder is twofold: first is the result of equal probability of occupying two different sites (sometimes referred to as positional or static disorder), and the second one originates from vibrational motion of coformer/solvent molecules, which can be visualized by the respective thermal ellipsoids (Figure 9). Both types of disorder may have an impact on the entropy contribution to the total energy of the studied systems.

a)

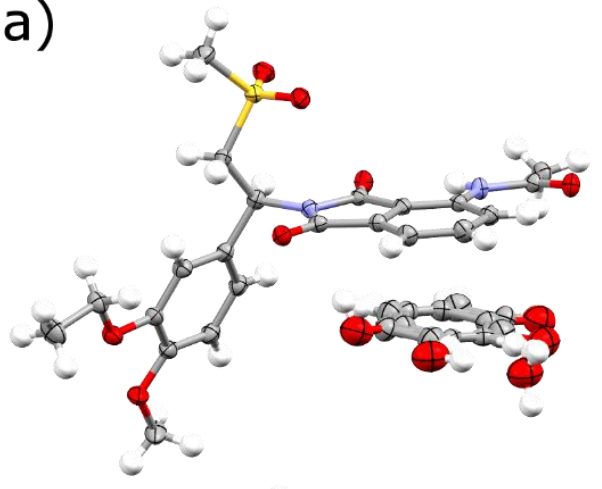

c)

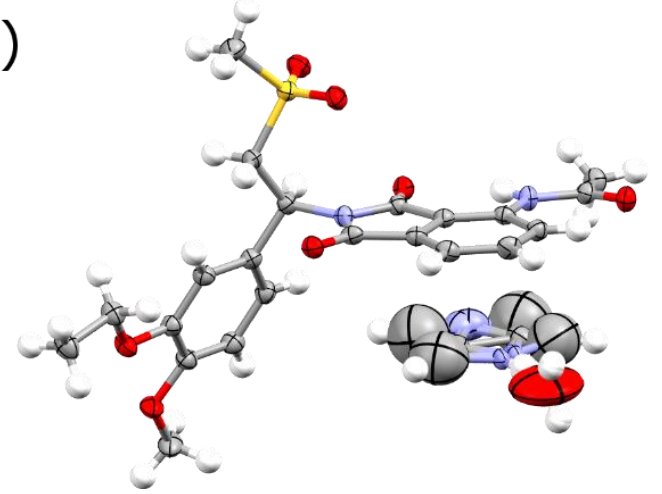

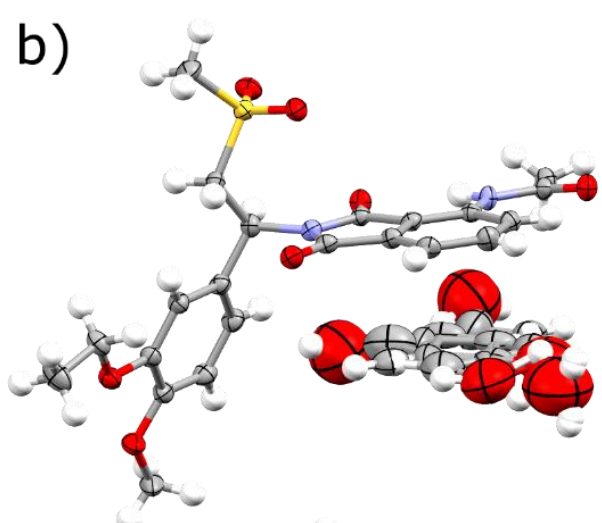

d)

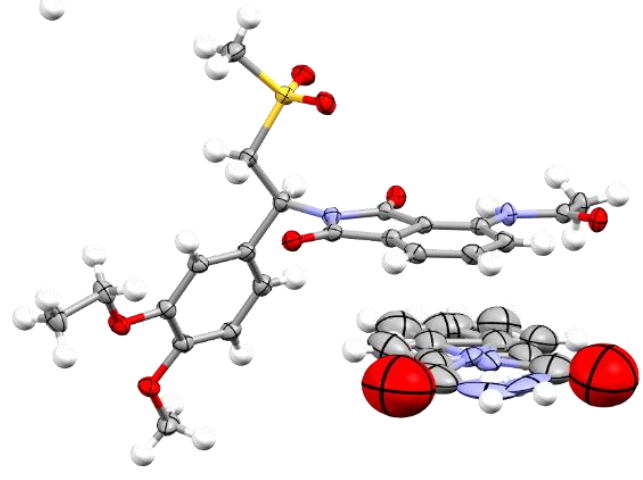

Figure 9. Thermal ellipsoids in the studied cocrystals, drawn at 50\% probability level.

In order to estimate this entropy contribution one can directly determine Gibbs free energy of formation of a cocrystal by measuring its solubility and comparing it with the solubility of physical mixture, according to the equation:

$$
\Delta G=-R T \ln \left(\frac{C_{A P R}^{p m}}{C_{A P R}^{c o c r}}\right)
$$

where R denotes the gas constant, T temperature of the measurement, while $C_{A P R}^{p m}$ and $C_{A P R}^{c o c r}$ are the concentration of apremilast from dissolution of a physical mixture and a cocrystal, respectively (Zhang et al., 2017; Ahuja et al., 2019). Since all enthalpies of formation of the 
studied cocrystals have positive values, negative Gibbs free energies indicate that the formation of cocrystals is indeed entropy driven. Such an experiment, however, can be easily performed only for the APR:PIC cocrystal, as in all other cases there is a water molecule present in the respective crystal structures, which is difficult to include in the solubility determination of a physical mixture, while possibly having significant influence on the experimental results. Therefore we decided to measure solubility only for the cocrystal and physical mixture of the APR:PIC system to gain at least an indication whether this above mentioned entropy term can indeed be responsible for the formation of APR cocrystals.

The obtained mean concentrations of APR dissolved from the physical mixture and the cocrystal were equal to $180.3 \pm 6.3$ and $171.2 \pm 5.8 \mathrm{mg} / \mathrm{ml}$ for $C_{A P R}^{p m}$ and $C_{A P R}^{c o c r}$, respectively, with the concentration for the physical mixture being always higher than for the cocrystal. This means that the free energy of cocrystal formation is negative and equal to $-0.132 \pm 0.002 \mathrm{~kJ} / \mathrm{mol}$ $(-0.29 \mathrm{~J} / \mathrm{g})$. As a result it may be concluded that the formation of APR:PIC cocrystals (and possibly also other APR cocrystals studied in this paper) is an entropy-driven process, with all probability associated with the observed disorder of the guest (coformer) molecules. Such an entropy-controlled formation of cocrystals has been already reported e.g. for celecoxibnicotinamid cocrystals (Zhang et al., 2017), and the entropy gain upon formation of cocrystals has been associated with an increase in molar volume of a crystal (higher molar volume means more space, which in turn means more freedom of movement, leading to higher entropy) (Perlovich, 2018). Here, such an increase in volume is not observed. Contrarily, molar volume upon formation of the studied cocrystals decreases, so it seems that it is rather a lack of strong directional hydrogen bonds between host and guest molecules that enables guest molecules to be disordered.

\section{Conclusions}

Four new cocrystals of APR, formed with aromatic coformers prone to act as hydrogen bonds donors, were found to be isostructural to the known cocrystals and solvates of APR, and held together mainly by aromatic - aromatic interactions, despite strong propensity of the guest molecules to form hydrogen bonds. The calculated energy of these aromatic - aromatic interactions is comparable to the energy of hydrogen bonds. Interestingly, calculated stabilization energies of these cocrystals were found to be positive in all cases, which means that their formation is associated with a loss of enthalpy. This loss has been linked to the strains in the conformation of APR after cocrystal formation. At the same time, solubility 
measurements indicated that the observed loss in enthalpy is compensated by the gain in entropy, so that the overall Gibbs free energy of the cocrystal formation was found to be negative. Therefore, the stabilizing role of disorder, observed in almost all isostructural cocrystals and solvates of APR discovered so far, can be postulated.

\section{Acknowledgements}

This study was financially supported by the National Science Center, Poland (Grant No. UMO2017/25/B/ST4/02684). The computational part of this work was carried out with a financial support of the Polish Ministry of Science and Higher Education, as a part of the fellowship 'Mobility Plus' (grant No 1664/MOB/V/2017/0). The Polish Infrastructure for Supporting Computational Science in the European Research Space (PL-GRID) and the IRIDIS High

Performance Computing Facility, with associated support services at the University of Southampton are gratefully acknowledged for providing computational resources. We are also grateful to the UK Materials and Molecular Modelling Hub for computational resources, which is partially funded by EPSRC (EP/P020194/1).

\section{References}

Ahuja, D., Svärd, M. \& Rasmuson, A. C. (2019). CrystEngComm 21, 2863-2874.

Althaus, S. M., Mao, K., Stringer, J. A., Kobayashi, T. \& Pruski, M. (2014). Solid State Nucl. Magn. Res. 57, 17-21.

Bacon, G. E. \& Jude, R. J. (1973). Z. Kristallog. 138, 19-40.

Bacon, G.E. \& Lisher, E. J. (1980). Acta Cryst. B36, 1908-1916.

Bolla, G. \& Nangia, A. (2016). Chem. Commun. 52, 8342-8360.

Brondel, N., Moynihan, E. J. A., Lehane, K. N., Eccles, K. S., Elcoate, C. J., Coles, S. J., Lawrence, S. E. \& Maguire, A. R. (2010). CrystEngComm 12, 2910-2927.

Case, D. H., Campbell, J. E., Bygrave, P. J. \& Day, G. M. (2016). J. Chem. Theory Comput. 12, 910-924. 
Clark, S. J., Segall, M. D., Pickard, C. J., Hasnip, P. J., Probert, M. J., Refson, K. \& Payne, M. C. (2005). Z. Kristallog. 220, 567-570.

Coombes, D. S., Price, S. L., Willock, D. J. \& Leslie, M. (1996). J. Phys. Chem. 100, 73527360.

Craven, B. M., McMullan, R. K., Bell, J. D. \& Freeman, H. C. (1977). Acta Cryst. B33, 25852589.

Dolomanov, O. V., Bourhis, L. J., Gildea, R. J., Howard, J. A. K. \& Puschmann, H. J. (2009). Appl. Cryst. 42, 339-341.

Douroumis, D., Ross, S. A. \& Nokhodchi, A. (2017). Adv. Drug Deliv. Rev. 117, 178-195.

Dudek, M. K., Kostrzewa, M., Paluch, P. \& Potrzebowski, M. J. (2018). Cryst. Growth Des. 18, 3959-3970.

Evora, A. O. L., Castro, R. A. E., Maria, T. M. R., Rosado, M. T. S., Silva, M. R., Canotilho, J. \& Eusebio, M. E. S. (2012). CrystEngComm 14, 8649-8657.

Frisch, M. J., Trucks, G. W., Schlegel, H. B., Scuseria, G. E., Robb, M. A., Cheeseman, J. R., Scalmani, G., Barone, V., Mennucci, B., Petersson, G. A., Nakatsuji, H., Caricato, M., Li, X., Hratchian, H. P., Izmaylov, A. F., Bloino, J., Zheng, G., Sonnenberg, J. L., Hada, M., Ehara, M., Toyota, K., Fukuda, R., Hasegawa, J., Ishida, M., Nakajima, T., Honda, Y., Kitao, O., Nakai, H., Vreven, T., Montgomery, J. A., Jr., Peralta, J. E., Ogliaro, F., Bearpark, M., Heyd, J. J., Brothers, E., Kudin, K. N., Staroverov, V. N., Kobayashi, R., Normand, J., Raghavachari, K., Rendell, A., Burant, J. C., Iyengar, S. S., Tomasi, J., Cossi, M., Rega, N., Millam, J. M., Klene, M., Knox, J. E., Cross, J. B., Bakken, V., Adamo, C., Jaramillo, J., Gomperts, R., Stratmann, R. E., Yazyev, O., Austin, A. J., Cammi, R., Pomelli, C., Ochterski, J. W., Martin, R. L., Morokuma, K., Zakrzewski, V. G., Voth, G. A., Salvador, P., Dannenberg, J. J., Dapprich, S., Daniels, A. D., Farkas, Ö., Foresman, J. B., Ortiz, J. V., Cioslowski, J. \& Fox, D. J. (2009) Gaussian 09, Revision D.01, Gaussian, Inc., Wallingford CT.

Goyal, P., Rani, D. \& Chadha, R. (2018). Cryst. Growth Des. 18, 105-118.

Grifasi, F., Chierotti, M. R., Gaglioti, K., Gobetto, R., Maini, L., Braga, D., Dichiarante, E. \& Curzi, M. (2015). Cryst. Growth Des. 15, 1939-1948.

Grimme, S. J. (2006). Comput. Chem. 27, 1787-1799. 
Karki, S., Friščić, T., Fabián, L., Laity, P. R., Day, G. M. \& Jones, W. (2009). Adv. Mater. 21, 3905-3909.

Kaur, R., Cavanagh, K. L., Rodríguez-Hornedo, N. \& Matzger, A. J. (2017). Cryst. Growth Des. 17, 5012-5016.

Kităgorodskiı̌, A. I. (1973). Molecular Crystals and Molecules, New York and London, Academic Press.

Lindeman, S. V., Shklover, V. E. \& Struchkov, Yu. T. (1981). Cryst. Struct. Commun. 10, 1173-1179,

Maartmann-Moe, K. (1966). Acta Cryst. 21, 979-982.

Mao, K., Wiench, W., Lin,V. S. Y. \& Pruski, M. (2009). J. Magn. Reson. 196, 92-95.

Mukherjee, A. \& Desiraju, G. R. (2014). Cryst. Growth Des. 14, 1375-1385.

Nielsen, N. C., Bildsøe, H., Jakobsen, H. J. \& Levitt, M. H. (1994). J. Chem. Phys. 101, 18051812.

Palusiak, M. \& Grabowski, S. J. (2002). J. Mol. Struct. 642, 97-104.

Patent No. EP 3339292 A1 (2018).

Patent No. US 9,850,205 B2 (2017).

Patent No. WO 2009/120167 A1 (2009).

Patent No. WO 2017/196192 A1 (2017).

Perlovich, G. L. (2018). CrystEngComm 20, 3634-3637.

Price, S. L., Leslie, M., Welch, G. W. A., Habgood, M., Price, L. S., Karamertzanis, P. G. \& Day, G. M. (2010). Phys. Chem. Chem. Phys. 12, 8478-8490.

Putra, O. D., Umeda, D., Nugraha, Y. P., Furuishi, T., Nagase, H., Fukuzawa, K., Uekusa, H. \& Yonemochi, E. (2017). CrystEngComm 19, 2614-2622.

Schafer, P. (2012). Biochem. Pharmacol. 83, 1583-1590.

Shakeel, F., Haq, N., Alanazi, F. K. \& Alsarra, I. A. (2017). Int. J. Pharm. 523, 410-417.

Sheldrick, G. M. (2015). Acta Cryst. C71, 3-8.

Stone, A. J. (2015). J. Chem. Theory Comput. 1, 1128-1132.

Taylor, C. R. \& Day, G. M. (2018). Cryst. Growth Des. 18, 892-904. 
Vasisht, K., Chadha, K., Karan, M., Bhalla, Y., Jena, A. K. \& Chadha, R. (2016). CrystEngComm 18, 1403-1415.

Wang, F.-Y., Zhang, Q., Zhang, Z., Gong, X., Wang, J.-R. \& Mei, X. (2018). CrystEngComm 20, 5945-5948.

Wicker, J. G. P., Crowley, L. M., Robshaw, O., Little, E. J., Stokes, S. P., Cooper, R. I. \& Lawrence, S. E. (2017). CrystEngComm 19, 5336-5340.

Wu, Y.-D., Liu, X.-H., Xu, J., Zhang, S.-H., Shen, K., Sun, L., He, Y.-M., Ma Y. \& Zhang, A.H. (2017a). Acta Cryst. E73, 821-824.

Wu, Y.-D., Zhang, X.-L., Liu, X.-H., Xu, J., Zhang, M., Shen, K., Zhang, S.-H., He, Y.-M., Ma Y. \& Zhang, A.-H. (2017b). Acta Cryst. C73, 305-313.

Yates, J. R., Pickard, C. J. \& Mauri, F. (2007). Phys. Rev. B76, 024401.

Yoshimura, M., Miyake, M., Kawato, T., Bando, M., Toda, M., Kato, Y., Fukami, T. \& Ozeki, T. (2017). Cryst. Growth Des. 17, 550-557.

Zhang, S.-W., Brunskill, A. P. J., Schwartz, E. \& Sun, S. (2017). Cryst. Growth Des. 17, $2836-2843$.

Zhu, Q., Shtukenberg, A. G., Carter, D. J., Yu, T.-Q., Yang, J., Chen, M., Raiteri, P., Oganov, A. R., Pokroy, B., Polishchuk, I., Bygrave, P. J., Day, G. M., Rohl, A. L., Tuckerman, M. E. \& Kahr, B. (2016). J. Am. Chem. Soc. 138, 4881-4889. 